\title{
Atividade Física para idosos: diretrizes para implantação de programas e ações
}

Physical Activity for the elderly: guidelines for the establishment of programs and actions

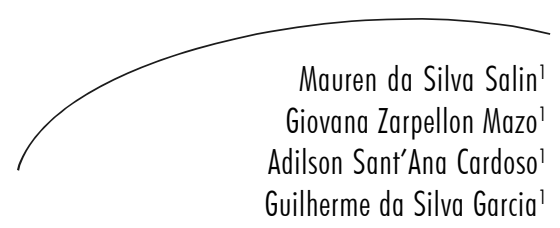

Resumo

O estudo objetivou descrever as diretrizes necessárias para a formulação de programas e ações de atividade física (AF) para idosos de grupos de convivência do município de São José, SC. Trata-se de pesquisa do tipo qualitativa, na qual foram entrevistados 116 idosos, 19 coordenadores de grupos, dois dirigentes administrativos do município e um representante do Conselho Estadual do Idoso (CEI) de Santa Catarina. Os sujeitos foram abordados com questões sobre conhecimento, participação, disponibilidade, necessidades, dificuldades e sugestões em relação aos atuais programas de atividade física (PAFs) oferecidos para a população idosa no município de São José, SC; o presidente do CEI foi indagado sobre a importância da implementação de programas e ações de AF para idosos, como parte das políticas públicas de promoção de saúde municipal. Também foram utilizados documentos, relatórios e projeto do Centro de Atenção à Terceira Idade (CATI) referentes ao atendimento à população idosa do município. Para o tratamento das informações, utilizou-se a técnica de análise de conteúdo. Diante das respostas, concluiu-se que para melhorar e ampliar os PAFs do município é necessário: descentralizar os PAFs; contratar profissionais de Educação Física; conscientizar sobre um envelhecimento ativo e os benefícios da prática regular de atividade física; estabelecer as parcerias com instituições públicas e privadas e divulgar o atendimento prestado.

\section{Abstract}

This study aimed to describe the guidelines for the formulation of physical activity programs and actions (PA) for elderly in convivial groups in the city of São José, SC. It is a qualitative research, where 116 seniors, 19 coordinators of groups, two leaders of the administrative council and a representative of the

Laboratório de Gerontologia - LAGER, Centro de Ciências da Saúde e do Esporte. Universidade do Estado de Santa Catarina. Florianópolis, SC, Brasil

Correspondência / Correspondence

Mauren da Silva Salin

Laboratório de Gerontologia - LAGER, Centro de Ciências da Saúde e do Esporte. Universidade do Estado de Santa Catarina

Rua Paschoal Simone 358, Coqueiros

88080350 - Florianópolis, SC - Brasil

E-mail: mauren@softin.com.br

Palavras-chave: Medicina esportiva. Atividade física. Programas de Bem-Estar Idoso. Centros de Convivência e Lazer. Políticas Públicas. 
State Council of the Elderly (SCE) from Santa Catarina State were interviewed. The subjects were approached with questions about knowledge, participation, availability, needs, problems and suggestions on current programs of physical activity (PPAs) offered to the elderly population in São José, SC; the president of the SCE was asked about the importance of implementing programs and actions of PA for the elderly as part of public policies to promote municipal health. We also used documents, reports and project of the Center for the Elderly Care (CEC) for the care of the elderly population of the municipality. For data processing, the content analysis technique was used. Based on the answers, we concluded that to improve and expand the PAPs of the municipality there must be: decentralization of the PPA; hiring Physical Education professionals; knowledge about active aging and the benefits of regular practice of physical activity; establishment of partnerships with public and private institutions; and disclosure of the delivered care.
Key words: Sports Medicine. Motor Activity. Health Promotion. Aged. Centers of Connivance and Leisure. Public Policies.

\section{INTRODUÇÃO}

O sedentarismo está evidente em todas as camadas sociais e faixas etárias atualmente, e tem como uma das causas os avanços dos recursos tecnológicos. $\mathrm{Na}$ velhice, o sedentarismo é em geral mais acentuado devido à crença popular de que, com o processo de envelhecimento, deve-se diminuir a prática de atividades físicas. ${ }^{1}$

A atividade física $(\mathrm{AF})$ regular surge como um meio de promoção de saúde e qualidade de vida. Em relação aos programas mundiais de promoção de saúde, os cientistas enfatizam cada vez mais a necessidade da AF como parte fundamental, pois neste novo milênio a inatividade física é considerada o principal problema de saúde pública. ${ }^{2,3}$ Existem evidências epidemiológicas que sustentam o efeito positivo de um estilo de vida ativo e/ou do envolvimento dos indivíduos em programas de exercícios físicos na prevenção e minimização dos efeitos deletérios do

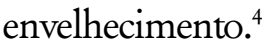

Nos últimos anos, houve aumento do interesse pela AF para os idosos, refletindo no crescente número de publicações científicas e reuniões/ eventos acadêmicos que focalizam essa temática. Também pode-se observar um aumento de programas de AF para idosos por todo mundo, como: "Pessoas saudáveis" (Inglaterra); "Viver ativo" (Canadá); "No Porto a vida é longa", "Mexase Mais - Atividade Física para Pessoas Idosas do
Conselho de Oeiras", "Coimbra Sênior" (Portugal); entre outros. Esses programas são oferecidos pelos órgãos governamentais locais e têm como objetivo promover a saúde dos idosos por meio da prática de $\mathrm{AF}^{5}$

No Brasil, apesar de existirem diferentes programas destinados à população idosa, observase que a implementação de programas de $\mathrm{AF}$ só é possível quando são averiguadas as características e necessidades dos idosos. Exemplo disto foi a análise feita por Benedetti ${ }^{5} \mathrm{em}$ pesquisa sobre o perfil dos idosos do município de Florianópolis. Nesse estudo, a pesquisadora concluiu que é possível planejar programas de AF que atendam às necessidades dos idosos, que têm garantido por lei, como consta no Estatuto do Idoso, ${ }^{6}$ capítulos IV e $\mathrm{V}$, o direito à saúde, ao esporte e ao lazer.

A falta de definição nas políticas públicas e de programas para a promoção do envelhecimento ativo e saudável faz com que frequentemente os profissionais da área gerontológica desenvolvam estratégias paliativas e descontinuadas, tais como atividades físicas sem planejamento ou ministradas por profissionais não capacitados e desqualificados para o trabalho com idosos.

Criar e fortalecer programas em geral, e especificamente de AF para idosos, exige investimentos de alocação de recursos que sejam aplicáveis à população idosa. Considerando a legislação vigente, muitos municípios brasileiros 
têm implantado programas de $\mathrm{AF}$ direcionados a esse público. O município de São José, SC tem investido na implantação desses programas, mas avaliações desses investimentos não têm ocorrido, e pouco se sabe sobre a percepção dos idosos com relação a esses programas. Desta forma, surge a necessidade de investigar essa realidade e avançar em propostas de diretrizes para a formulação de programas e ações de $\mathrm{AF}$ para idosos, como parte das políticas públicas de promoção de saúde do município de São José, SC. Assim, o presente estudo objetivou descrever as diretrizes necessárias para a formulação de programas e ações de atividade física (AF) para idosos de grupos de convivência do município de São José, SC.

\section{METODOLOGIA}

\section{População e amostra}

A população deste estudo foi constituída por 262 idosos e 43 coordenadores participantes de grupos de convivência de São José, SC, que fizeram parte do Projeto de Mazo, ${ }^{7}$ que descreve a rede de atendimento ao idoso e o nível de atividade física dos idosos de grupos de convivência do município de São José - SC.

Para a amostra foram selecionados os idosos e coordenadores que participaram da pesquisa de $\mathrm{Mazo}^{7}$, sendo que os critérios de exclusão foram: idosos que não participavam mais dos grupos de convivência, não possuíam telefone, não residiam mais no município, que estavam doentes no período da coleta (junho a agosto de 2006) ou faleceram. Os coordenadores foram os que compareceram à reunião da Associação de Voluntárias Josefense da Ação Social - AVJAS, agendada para a aplicação desta pesquisa. Deste modo, a amostra do estudo foi constituída por 116 idosos participantes de grupos de convivência do município de São José, SC, e 19 coordenadores dos grupos.

Também foram entrevistados o diretor do Departamento da Terceira Idade e a coordenadora do Centro de Atenção à Terceira idade (CATI), do Município de São José e o presidente do Conselho Estadual do Idoso (CEI), de Santa Catarina, gestão 2003/2006.

Instrumentos de coleta de dados

Foram utilizados como fontes de pesquisa o banco de dados da pesquisa de $\mathrm{Mazo}^{7}$ e documentos, relatórios e o projeto do CATI, referentes ao atendimento à população idosa do município de São José, SC.

Também foram aplicadas entrevistas com questões abertas sobre conhecimento, participação, disponibilidade, necessidades, dificuldades e sugestões dos idosos, coordenadores dos grupos de convivência e dirigentes administrativos em relação aos atuais programas de atividade física (PAFs) para a população idosa no município de São José, SC; o presidente do CEI foi indagado sobre a importância da implementação de programas e ações de AF para idosos, como parte das políticas públicas de promoção de saúde municipal.

\section{Procedimentos de coleta de dados}

A pesquisa foi aprovada pelo Comitê de Ética da UDESC em 29/03/2005, processo $n^{\circ} 164 /$ 2005. Foram coletadas do banco de dados da pesquisa de $\mathrm{Mazo}^{7}$ as informações quanto à rede de atendimento comunitário existente para os idosos do município, principalmente as específicas sobre os PAFs e os dados de identificação e a presença de doenças nos idosos.

Para a execução das entrevistas, foi realizado inicialmente um contato pessoal ou telefônico com os sujeitos do estudo, a fim de esclarecer os objetivos da pesquisa, o sigilo de identificação, o modo de aplicação, o destino dos dados e solicitar a participação. Após, foi agendada a entrevista. O termo de consentimento livre e esclarecido foi assinado pelos idosos durante a realização da pesquisa de Mazo, ${ }^{7}$ informando que futuramente eles seriam contatados por telefone ou pessoalmente para novos questionamentos a respeito de sua participação no CATI. 
Também foi realizado um estudo piloto com dez idosas participantes de grupos de convivência de São José, SC, que foram entrevistadas para verificar a clareza nas perguntas e a viabilidade de aplicação do instrumento por telefone. Todas as idosas entrevistadas manifestaram facilidade/ clareza para responder às perguntas da pesquisa por telefone. Deste modo, as entrevistas com os idosos o estudo foram realizadas por telefone.

Os coordenadores foram entrevistados pessoalmente pela pesquisadora responsável, após assinarem o termo de consentimento livre e esclarecido de participação na pesquisa, durante uma reunião da AVJAS. O diretor do Departamento da Terceira Idade e a coordenadora do CATI foram entrevistados pessoalmente na sede do CATI. O presidente do CEI de Santa Catarina foi entrevistado via e-mail, por solicitação do mesmo.
Tratamento dos dados

Os dados quantitativos foram tabulados no pacote estatístico SPSS versão 13.0 e tratados mediante estatística descritiva (frequência simples e percentagem).

As entrevistas foram transcritas e lidas na íntegra, para o tratamento das informações pela técnica de análise de conteúdo, ${ }^{8}$ aglutinando-se as unidades de sentido e estabelecendo as categorias e subcategorias que constituíram os eixos temáticos para a análise, seguindo-se as etapas operacionais: (a) constituição do corpus; (b) composição das unidades de análise; (c) categorização; (d) quantificação das categorias. Os dados qualitativos foram tabulados e armazenados no software QSR-NUD*IST (Qualitative Research and Solutions), para a análise de conteúdo, conforme o organograma apresentado na figura 1.

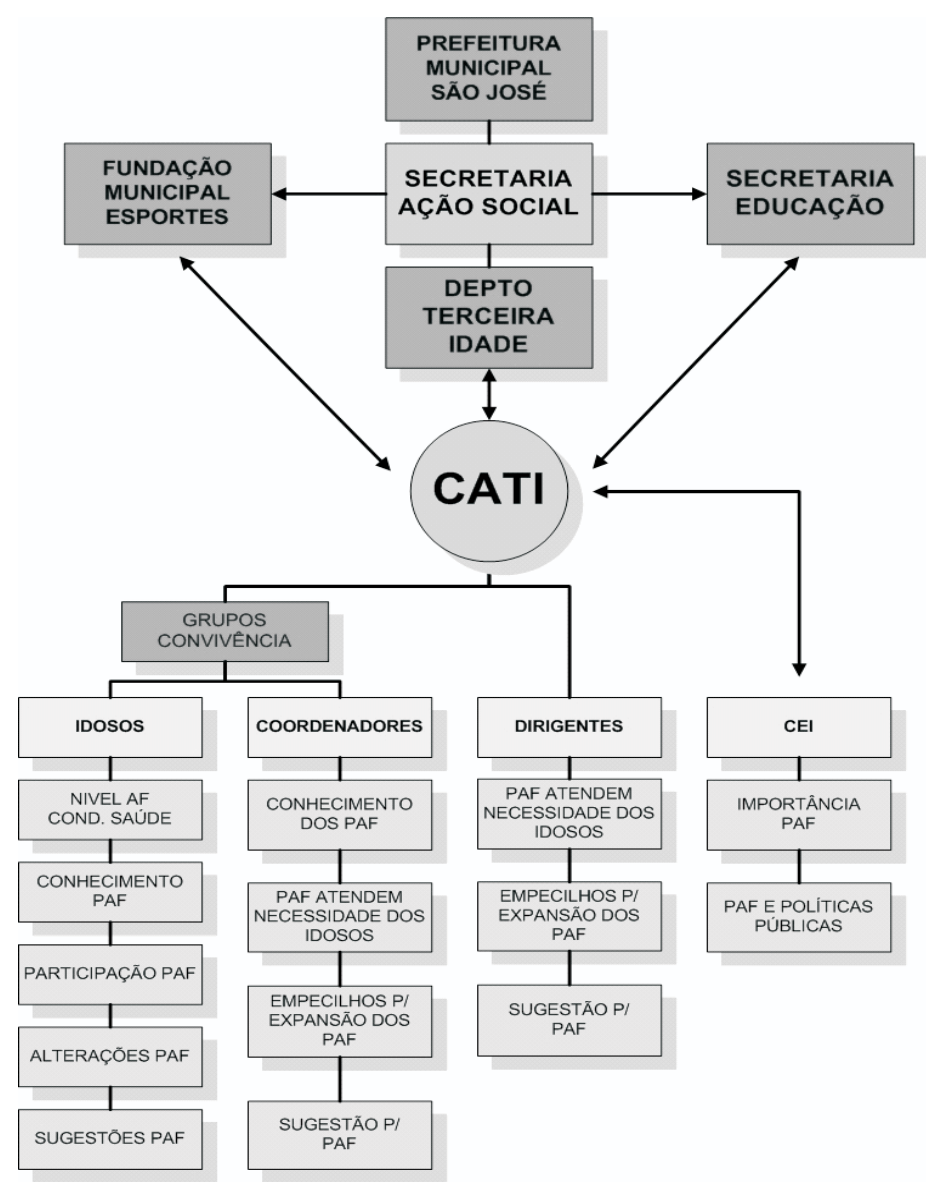

Figura 1 - Organograma com a rede de atendimento da PMSJ e as categorias de análise de conteúdo dos depoimentos dos sujeitos do estudo (PMSJ: Prefeitura Municipal de São José; CATI: Centro de Atenção à Terceira Idade; PAF: Programas de Atividade Física) 


\section{RESULTADOS E DISCUSSÃO}

As modalidades disponíveis atualmente no CATI são a ginástica do programa "Mexa-se", em convênio com a Secretaria Municipal de Educação e com um shopping da cidade, a ginástica do programa "Exercita-se", uma parceria do CATI com a Fundação Municipal de Esportes e, as aulas de ioga, tai chi chuan, dança sênior e dança folclórica oferecidas pelo CATI.

De acordocom a figura 2, dos idosos entrevistados, 76,7\% declararam nãoter conhecimento sobre nenhum PAF oferecido pelo CATI. Dos coordenadores entrevistados, $84 \%$ declararam ter conhecimento, mas desconhecem as modalidades disponíveis.

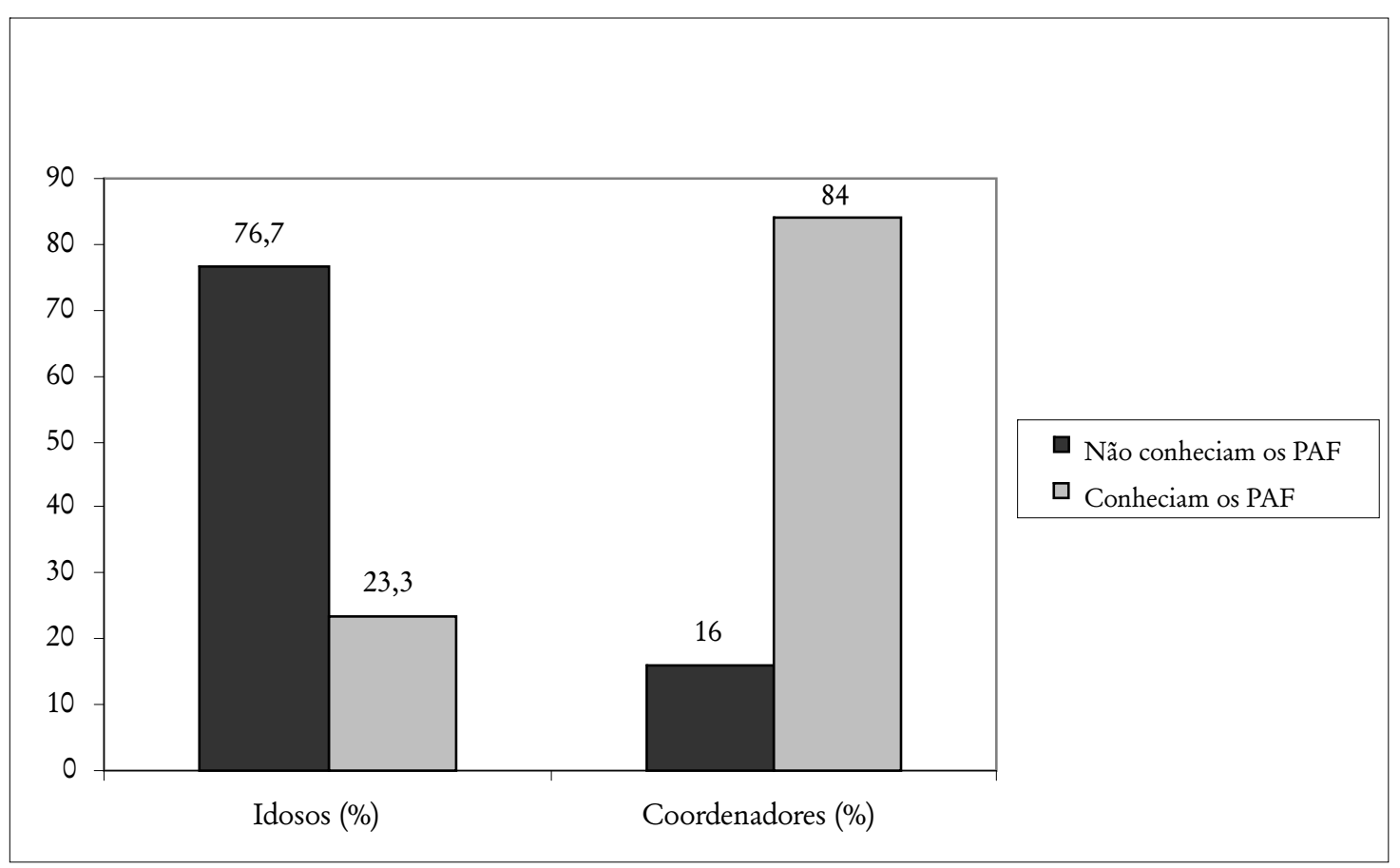

Figura 2 - Conhecimento sobre os PAF do CATI. Florianópolis, SC, jun/ago.2006.

Observa-se que a maioria dos idosos e coordenadores entrevistados desconhece as atividades oferecidas no CATI. Ainda que os idosos e coordenadores participem das atividades mensais desenvolvidas pelo centro e os coordenadores participem das reuniões da AVJAS, constata-se a necessidade de formas mais eficazes de passar as informações sobre os PAFs desenvolvidos no CATI e em outras secretarias.

Para Sociedade Brasileira de Medicina do Esporte e para a Sociedade Brasileira de Geriatria e Gerontologia, a AF deve ser incentivada e estimulada para os indivíduos idosos, inclusive através de iniciativas públicas e privadas, pois se torna um excelente instrumento de promoção de saúde para essa população. ${ }^{9}$
Para que as pessoas idosas pratiquem $\mathrm{AF}$ regularmente, elas devem ter conhecimento sobre seus benefícios, superar vícios e comportamentos inadequados e desenvolver hábitos propícios à prática. ${ }^{1}$ Entretanto, um nível adequado de conhecimento sobre os PAFs não necessariamente reflete um envolvimento regular nas práticas. A promoção de saúde por meio de AF regulares deverá enfatizar, além do incremento ao conhecimento, estratégias para superar as barreiras, facilitando assim a adoção de um estilo de vida ativo. $^{10}$

Os idosos e os coordenadores também foram indagados sobre como tomaram conhecimento dos PAFs oferecidos pelo município, podendo ser observados os resultados encontrados na figura 3 . 


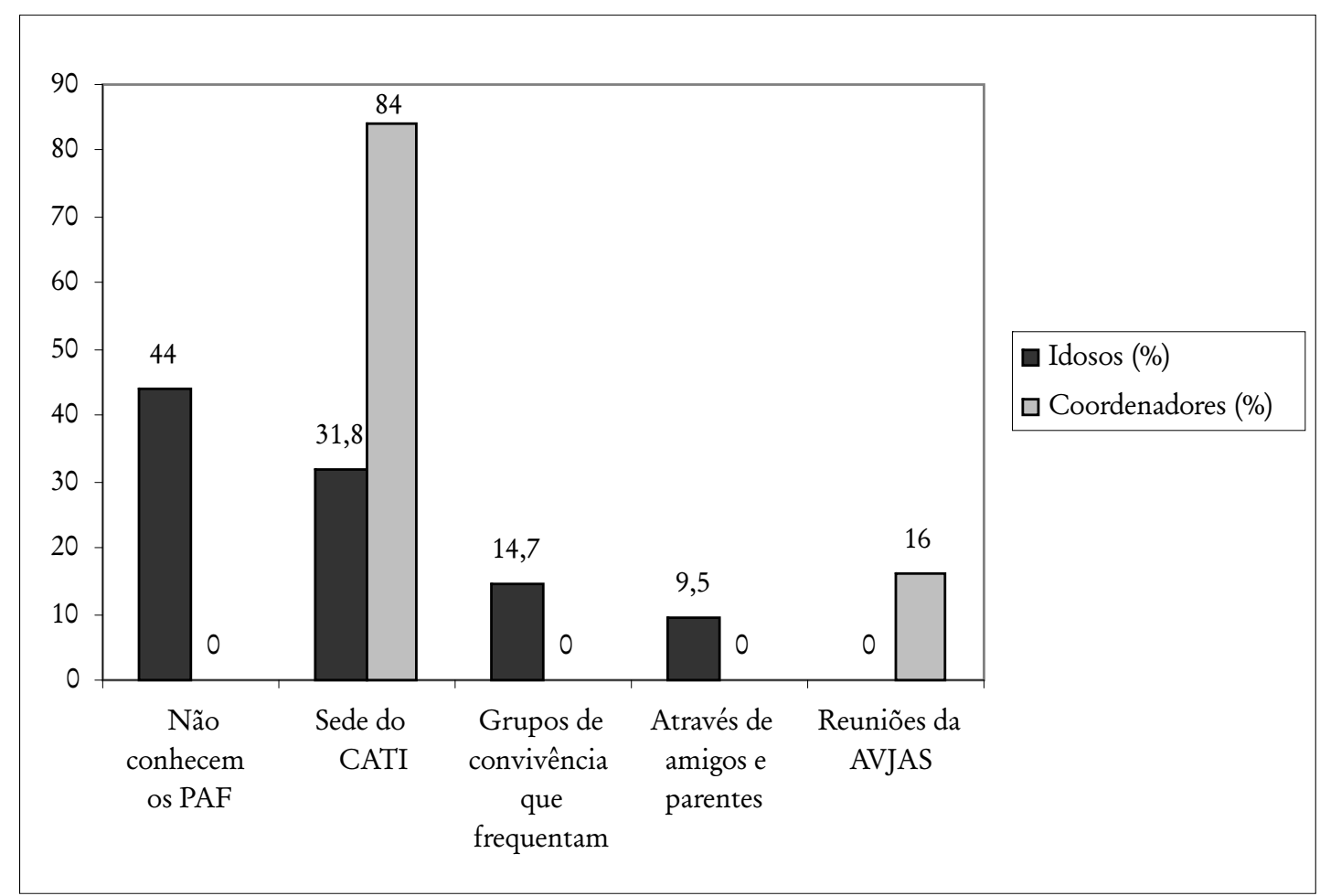

Figura 3 - Como tomaram conhecimento sobre os PAF do município. Florianópolis, SC, jun/ago.2006.

O papel dos profissionais da área da saúde tem grande relevância no que diz respeito ao incentivo aos idosos para prática de $\mathrm{AF}$, como foi mencionado por alguns idosos durante as entrevistas, referindo-se ao geriatra que atende na Policlínica. É função dos profissionais da saúde, educadores, líderes comunitários e políticos minimizarem as barreiras e ampliarem em quantidade e diversidade os fatores facilitadores de adesão às práticas de AF. É por meio da comunicação que chegarão até os idosos as informações pertinentes aos benefícios dos PAFs para um envelhecimento saudável. Segundo Gobbi, ${ }^{11}$ difundir a prática de exercícios físicos entre os idosos é uma estratégia simples, barata e eficaz, tanto para diminuir os custos relacionados à saúde, quanto para melhorar a qualidade de vida. Programas que visam ao envelhecimento ativo são relevantes para o sucesso econômico de países que, como o Brasil, enfrentam acentuado processo de envelhecimento, o que indica uma crescente procura por serviços, sobretudo na área da saúde pública. ${ }^{12}$
Para que os objetivos propostos pelo CATI sejam concretizados, se faz necessária uma dinâmica na divulgação dos PAFs por ele oferecidos, de maneira que os idosos possam decidir ingressar ou não nos programas. Farinatti $\&$ Ferreira ${ }^{13}$ destacam, através de estudos, que as redes de suporte social facilitam a disseminação de informações, o que encoraja a realização de desejos individuais, pois as mesmas proporcionam companhia, segurança e ajuda, o que é essencial à promoção de saúde de grupos particularmente vulneráveis à solidão, como é o caso dos idosos.

A maioria dos idosos entrevistados, $81,9 \%$, não participa de nenhum programa. Esse é um dado preocupante, visto que a participação em PAFs regulares e moderadas pode retardar declínios funcionais, além de diminuir o aparecimento de doenças crônicas em idosos saudáveis ou doentes crônicos. ${ }^{14}$ Os motivos pelos quais os idosos não participam dos PAFs do município podem ser observados na figura 3 . 


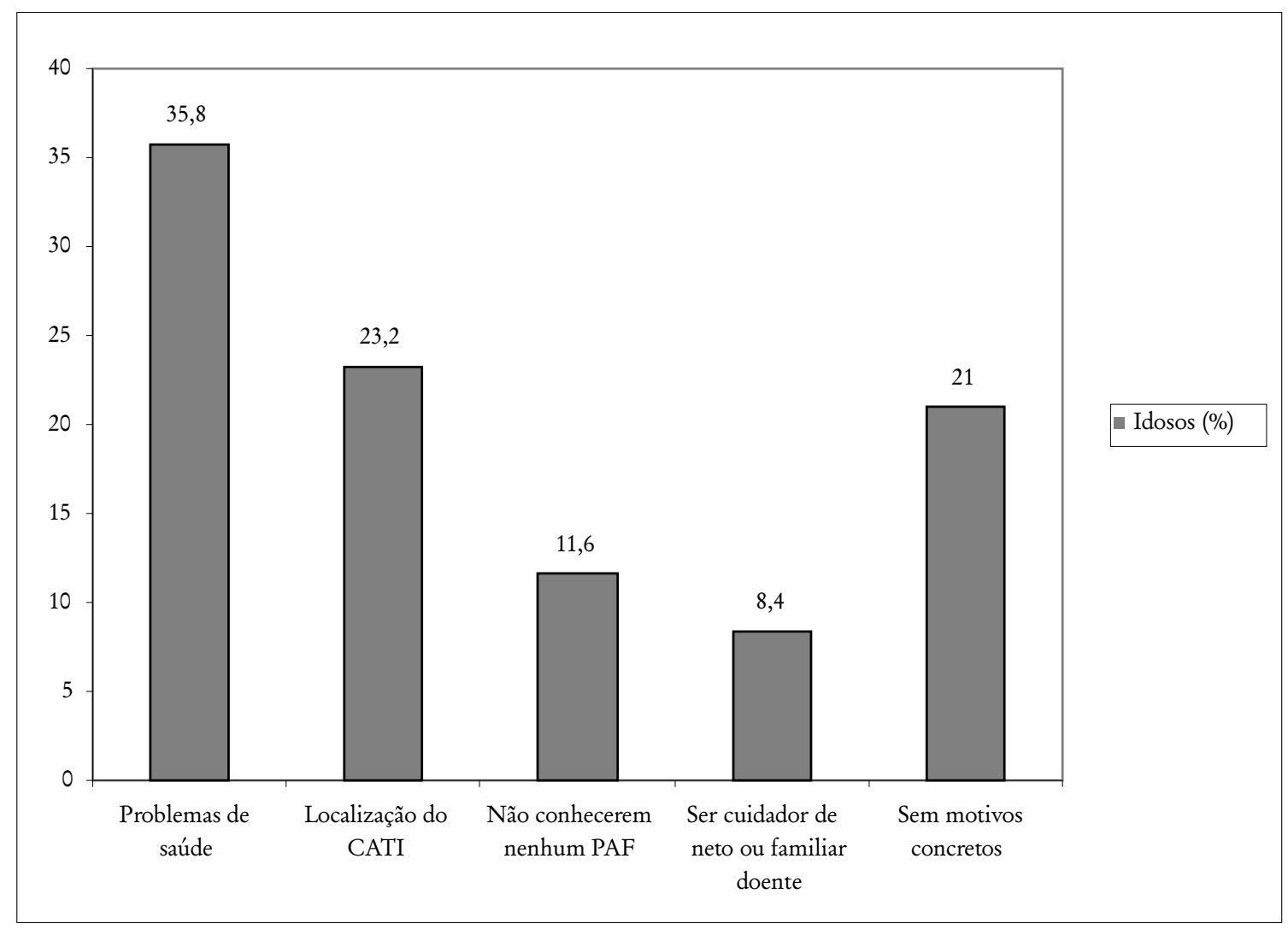

Figura 4 - Motivos para não participar em PAF. Florianópolis, SC, jun/ago.2006.

Estudo realizado por Matsudo et. al. ${ }^{10}$ verificou que realmente existem algumas barreiras que impedem o acesso dos idosos à prática de $\mathrm{AF}$, entre as quais se encontram a falta de equipamentos e de local para prática, o medo de lesão e a falta de conhecimento. Segundo os autores, essas barreiras poderiam ser superadas com a divulgação de novas mensagens de promoção da $\mathrm{AF}$, que mostram que não há necessidade de equipamentos, local ou conhecimento para uma pessoa ser regularmente ativa.

Um dos principais motivos para os idosos não participarem dos PAFs foram os problemas de saúde. Apesar de o processo de envelhecimento aumentar a probabilidade de desenvolver certas doenças, é importante esclarecer que envelhecer não é sinônimo de adoecer, especialmente quando as pessoas desenvolvem hábitos de vida saudáveis. ${ }^{15} \mathrm{~A}$ AF praticada de forma sistemática diminui a intensidade e a velocidade de implantação de disfunções musculoesqueléticas, neuromusculares e cardiopulmonares decorrentes do envelhecimento do organismo. ${ }^{16}$
Para o American College os Sports Medicine, ${ }^{17}$ a inatividade física constitui uns dos riscos para o desenvolvimento tanto de doenças cardiorrespiratórias como musculoesqueléticas. A probabilidade de as pessoas desenvolverem doenças crônicodegenerativas aumenta com o processo de envelhecimento, mas a frequência em $\mathrm{AF}$ pode resultar na redução do risco de doenças cardíacas ou outras doenças. ${ }^{14}$

Diante dos outros motivos apresentados pelos idosos para não participarem dos $\mathrm{PAF}$, verificamse em sua maioria fatores externos, que não justificam a ausência total desses idosos nos programas. A maior parte dos idosos reúne as condições necessárias uma vida autônoma e produtiva, sem apresentar sintomas de decadência mental ou física, que, por meio de acesso ao conhecimento, poderia contribuir para o engajamento das pessoas idosas em atividades físicas. ${ }^{13}$

Embora o CATI disponibilize alguns PAFs, verifica-se que o número de participantes ainda é 
mínimo, comparado com a população total de idosos cadastrados no centro. Além dos motivos mencionados pelos idosos para não participarem dos PAFs, é preciso verificar as barreiras que impedem o acesso dos idosos aos programas.

Sobre as necessidades dos idosos estarem sendo atendidas pelos PAFs oferecidos pelo município, $79 \%$ dos coordenadores declaram que não, pois para eles os PAFs ofertados pelo município atualmente não atendem às necessidades dos idosos. Para eles são oferecidos poucos programas e faltam profissionais para atender à demanda.

Quando perguntados se os PAFs estariam atendendo ou não às necessidades dos idosos do município, os dirigentes declararam acreditar que os programas atendem grande parte das necessidades, mas apenas para os idosos que frequentam o CATI regularmente. Eles também salientaram que o CATI ainda não está pronto, sendo esperados móveis, materiais, a conclusão das obras da piscina, bem como a contratação de profissionais capacitados para atender aos idosos.

Para atender às necessidades dos idosos, Abreu ${ }^{19}$ coloca que a implementação de programas e ações de AF não deve ser encarada como algo segmentado, mas transdisciplinar, que deve estar presente em diversas políticas públicas e, pela condição de vulnerabilidade do segmento idoso, também possuir relativa importância na área da saúde.

Segundo Paschoal, ${ }^{20}$ se os indivíduos envelhecerem com autonomia e independência, com boa saúde física, desempenhando papéis sociais, permanecendo ativos, a qualidade de sua vida pode ser muito boa. Veras e Caldas ${ }^{21}$ estabelecem uma ligação entre saúde e qualidade de vida e enfatizam a criação de ambientes favoráveis à saúde e ao desenvolvimento de habilidades pessoais, onde possam ser inseridas ações de promoção de saúde que ampliam o âmbito das intervenções com os indivíduos idosos. Os programas de AF destinados ao público idoso devem atender a suas necessidades específicas, em seus aspectos fisiológicos, psicológicos e sociais, assim como devem ser conhecidos o perfil e a realidade social desse público.
Ao serem indagados sobre a existência de dificuldades para expansão dos PAFs, 53\% dos coordenadores entrevistados declararam não existir nenhum tipo de dificuldade para sua expansão. Entretanto, $47 \%$ declararam que existem dificuldades políticas e financeiras. Os dirigentes também acreditam que existam dificuldades financeiras e políticas, mas destacam as políticas, porque ocasionam a demora ou impedem a realização de determinadas ações pertinentes ao bom desempenho nas atividades propostas pelo CATI.

Essas dificuldades não se justificam para o Conselho Estadual do Idoso, visto que está previsto em lei, como consta no Estatuto do Idoso $^{6}$, artigo 20 , o direito do idoso ao esporte e ao lazer, o que pode ser exigido do governo mediante ação para defesa desses direitos, consoante art. 82 do mesmo Estatuto. ${ }^{19}$

Estudo realizado por Benedetti ${ }^{22}$ em algumas cidades da Europa constatou que todos os programas de AF para idosos estudados são vinculados à Secretaria da Assistência Social ou do Desporto das prefeituras municipais, como parte de ações de políticas públicas de lazer e saúde. As prefeituras oferecem programas de atividade física para os idosos devido a sua alta demanda. No início, os programas eram oferecidos de modo assistemático, apenas como ocupação do tempo livre. Com o passar do tempo, os idosos começaram exigir que as prefeituras municipais os oferecessem regularmente.

Portanto, existem as leis que asseguram os direitos dos cidadãos idosos, como a Política Nacional do Idoso, o Estatuto do Idoso e a Lei Estadual e Municipal que dispõe sobre a Política Estadual e Municipal do Idoso. Essas leis estabelecem mecanismos, instrumentos e orientações que facilitam a coordenação de políticas, participação popular em organizações representativas, com justiça, conhecimentos oportunos, visando a uma saudável democracia participativa para uma melhor qualidade de vida dos cidadãos idosos. ${ }^{23}$ Além disso, informações sobre o envelhecimento da população, como indicadores de saúde, são necessários, uma vez que 
contribuem para a elaboração de estratégias e estabelecimento de metas para programas específicos direcionados à população que envelhece. ${ }^{24}$

Uma das grandes dificuldades para a expansão dos PAFs sem dúvida seria a falta de profissionais capacitados para atender ao público em questão, mas que facilmente poderia ser resolvida, pois segundo Medeiros e Osório, ${ }^{25}$ a Política Nacional do Idoso prevê a possibilidade de articulação de entidades governamentais com organizações nãogovernamentais para viabilizar a capacitação de recursos humanos. As premissas do próprio CATI preveem parcerias com órgãos e entidades públicas e privadas, bem como a utilização de estagiários para operacionalização das atividades. ${ }^{26}$

As sugestões para melhorar ou implementar os PAFs na opinião dos idosos entrevistados podem ser observadas na figura 5.

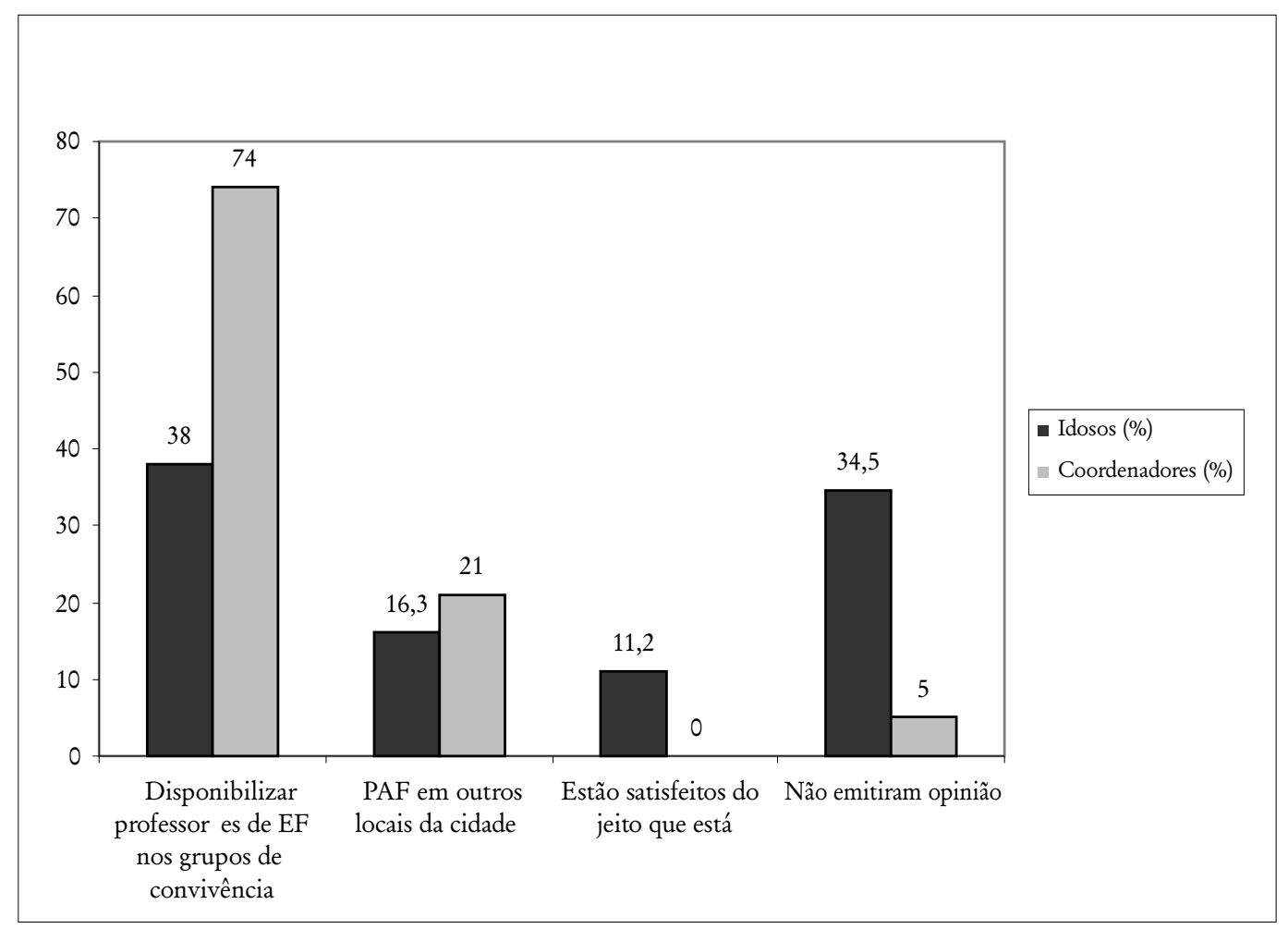

Figura 5 - Sugestões para melhorar ou implementar os PAF. Florianópolis, SC, jun/ago.2006.

Verifica-se que a sugestão para que a administração pública municipal disponibilize profissionais da área de educação física para ministrar os PAFs diretamente nos grupos é comum entre os idosos e coordenadores dos grupos de convivência. Também se observou, durante as entrevistas, o descontentamento dos idosos e coordenadores com relação ao fato de ter sido disponibilizada no passado uma professora de educação física para atender aos grupos e esse benefício ter sido posteriormente cancelado pela administração pública municipal.

No decorrer da entrevistas, verificou-se que para os idosos é extremamente importante a presença de um profissional da área de educação física na sede dos grupos. Para eles, o acompanhamento de um professor é uma forma de incentivo tanto para praticar as atividades como para participar dos encontros do grupo. Outra sugestão comum aos idosos e coordenadores foi a descentralização dos PAFs, ou seja, disponibilizar esses programas em outros locais na cidade além dos já oferecidos no CATI.

De acordo com as sugestões de descentralizar as atividades, disponibilizando-as em vários locais da cidade, toma-se como exemplo o Projeto "Mexa-se pela Saúde na Terceira Idade", no 
município de Florianópolis, que abrange 28 comunidades, atendendo a 1.233 idosos, distribuídos em 45 grupos, sendo: 10 grupos de ginástica, com atividades físicas e desportivas; 03 instituições asilares de assistência social do município, com atividades físicas, recreativas e de lazer; 01 grupo de dança; e 31 grupos de convivência para idosos, com atividades recreativas e de lazer. ${ }^{27}$

Aos dirigentes também foi solicitado que expusessem sugestões para melhorar ou implementar o PAF para os idosos do município. De comum acordo, eles responderam que deveria ser incentivada a criação de mais grupos de convivência na cidade para que, dessa forma, mais idosos possam participar dos programas do CATI.

É dever do Estado, através de políticas e programas, estimular pessoas inativas a se tornarem mais ativas à medida que envelhecem, sendo particularmente importante providenciar áreas seguras para caminhadas e apoiar atividades comunitárias culturalmente apropriadas que estimulem a atividade física e que sejam organizadas e lideradas pelos próprios idosos. $\mathrm{O}$ conselho de profissionais, orientando o idoso para a participação gradativa nessas atividades e programas de reabilitação física que ajudem as pessoas idosas a se recuperarem de problemas de mobilidade, é eficiente. ${ }^{14}$

\section{CONSIDERAÇÕES FINAIS E RECOMENDAÇÕES}

A partir dos resultados encontrados sobre a rede de atendimento aos idosos do município de São José, sobre o nível de AF da população em questão, e diante dos depoimentos dos idosos, coordenadores e representantes $\mathrm{da}$ administração pública municipal, bem como as contribuições do CEI, foi possível elaborar sugestões para a implantação de programas e ações de AF, com vistas à promoção de saúde dos idosos. São elas:

1. a descentralização das AF para os idosos, ou seja, disponibilizar PAFs em associações, clubes, salões de igreja, centros comunitários ou outros locais que permitam reunir várias pessoas e contemplem os idosos que não têm a oportunidade de frequentar o CATI;

2. a contratação de profissionais de Educação Física, capacitados para ministrar programas de AF para idosos, no mínimo três vezes por semana, nos grupos de convivência,

3. a conscientização dos idosos e da comunidade em geral sobre o envelhecimento ativo e benefícios da prática regular de AF;

4. congregar a atuação das diferentes secretarias municipais com ações voltadas aos PAFs direcionadas aos idosos;

5. estabelecer parcerias com instituições públicas ou privadas, como universidades, associações de moradores, clubes, centros sociais, entre outros;

6. implementar as premissas previstas pelo CATI: ofertas de serviço de saúde com foco educativo e preventivo e participação de estagiários para operacionalização das atividades;

7. divulgar o atendimento prestado ao público idoso do município para a comunidade em geral;

8. implementar atividades de lazer ativo que envolvam a comunidade, como passeios de bicicleta, caminhadas, trilhas ecológicas.

No presente estudo, constatou-se que a rede de atendimento aos idosos no município de São José está se estabelecendo com o CATI. O município apresenta vários projetos e programas voltados ao idoso, mas percebe-se que existe uma carência no que diz respeito aos PAFs como meio de promoção de saúde. Sendo assim, espera-se que este estudo sirva como contribuição para aprimorar o atendimento aos idosos do município. 


\section{REFERÊNCIAS}

1. Mazo GZ, Lopes MA, Benedetti TRB. Atividade física e o idoso: concepção gerontológica. 2 ed. Porto Alegre: Sulina, 2004; 247p.

2. Matsudo SM. Envelhecimento e atividade física. Londrina: Midiograf, 2001; 195p.

3. Blair SN. Physical Inactivity: the major public health problem of the new millennium the evidence and strategies for change.

In:Caderno de Resumo do congresso desporto, atividade física e saúde: o contributo da ciência e o papel da escola. Porto: FCDEF 2002; p.2-4

4. American College of Sports Medicine. Teste de esforço e prescrição de exercício. 5 ed. Rio de Janeiro: Revinter ; 2000.

5. Benedetti TRB. Atividade Física: uma perspectiva de promoção da saúde do idoso no município de Florianópolis. Florianópolis.

Tese - Universidade Federal de Santa Catarina; 2004.

6. Brasil.Lei $\mathrm{n}^{\circ}$ 10.741 Out 2003.Estatuto do Idoso.[Acesso em 20 Jan 2010]. Disponível em: http://www.planalto.gov.br/ccivil/LEIS/2003/ L10.741.htm

7. Mazo GZ. Relatório com o banco de dados da pesquisa: rede de atendimento e nível de atividade física dos idosos de grupos de Convivência do município de São José - SC. Florianópolis: CEFID/UDESC; 2005.

8. Bardin L. Análise de conteúdo. Lisboa: Edições 70; 1997.

9. Nóbrega ACL, et al. Posicionamento oficial da Sociedade Brasileira de Medicina do Esporte e da Sociedade Brasileira de Geriatria e Gerontologia: atividade física e saúde no idoso. Rev Bras Med Esporte 1999 nov./dez; 5(6): 207 211.

10. Matsudo SM, Matsudo VKR, Barros Neto TL. Atividade Física e envelhecimento: aspectos epidemiológicos. Rev. Bras Med Esporte 2001 jan./fev; 7(1).

11. Goobi S. Atividade física para pessoas idosas e recomendações da Organização Mundial de Saúde de 1996. Rev. Bras Ativ Fis Saúde 1997; 2(2): 41-49.

12. Mazo GZ, Cardoso AS, Dias RG, Balbé GP, Virtuoso JF. Do diagnóstico à ação: grupo de estudos da terceira idade: alternativa para a promoção do envelhecimento ativo. Rev Bras Ativ Fis Saúde 2009; 14(1).
13. Farinatti PTV, Ferreira MS. Saúde, promoção da saúde e educação física: conceitos, princípios e aplicações. Rio de Janeiro: EDUERJ; 2006. 288p.

14. World Health Organization. Envelhecimento ativo: uma política de saúde. Brasília: Organização PanAmericana da Saúde; 2005.

15. Neri AL. Envelhecer bem no trabalho: possibilidades individuais, organizacionais e sociais. São Paulo : SESC .Rev A Terceira Idade 2002; 13(24): 7-27.

16. Caromano FA, Kerbauy RR. Efeitos do treinamento e da manutenção de atividade física em quatro idosos sedentários saudáveis. Rev Bras Fisioter 2001 ago./dez; 8(2): 72-80.

17. American College of Sports Medicine. Recursos do ACSM para o personal trainer. Rio de Janeiro: Guanabara Koogan; 2006.

18. Minayo MCS, Hartz ZMA, Buss PM. Qualidade de vida e saúde: um debate necessário. Rev. Ciênci Saúde Coletiva 2000; 5(1): 7-18.

19. Abreu H. Publicação on -line [mensagem pessoal]. Mensagem recebida por mauren@softin.com.br em 28 set 2006.

20. Paschoal SMP. Qualidade de vida do idoso: elaboração de um instrumento que previlegia sua opinião. São Paulo. Dissertação [Mestrado em Medicina] —Universidade de São Paulo; 2000.

21. Veras RP, Caldas CP. Promovendo a saúde e a cidadania do idoso: o movimento das universidades da terceira idade. Rev Ciênc Saúde Coletiva 2004; 9(2): 423-432.

22. Benedetti TRB, Gonçalves LHT, Mota JÁPS. Uma proposta de política pública de atividade física para idosos. Rev Texto Contexto Enferm 2007; 16(3): 387-398.

23. Prefeitura Municipal de Florianópolis. Leis dos Idosos: municipal, estadual e Federal .

Florianópolis :Secretaria Municipal da Habitação, Trabalho e Desenvolvimento Social; 2004.

24. Rebouças M, Galera SC, Pereira SRM, Pereira MG. Que informações são necessárias para o conhecimento da situação de saúde do idoso? Rev Ciênc e Saúde Coletiva 2008; 13(4): 13051312.

25. Medeiros M, Osório R. Políticas de assistência ao Idoso no Brasil. In: Como Vai? População brasileira. Brasília: IPEA . 
Ago 1999 [Acesso em 15 Out Jun].

Disponível em http://www.ipea.gov.br/pub/ comovai/comovai0299.pdf. Acesso em 15/ $10 / 06$.

26. Gomes RT, Verani DN, Ancora M. Projeto: centro de atenção à Terceira Idade. Tubarão:
Prefeitura Municipal de São José/SC e UNISUL; 2005.

27. Prefeitura Municipal de Florianópolis. Programa de atenção à Terceira Idade. Florianópolis :Secretaria de Habitação, Trabalho e Desenvolvimento Social; 2004.

Recebido: 08/9/2009

Revisado: $18 / 5 / 2010$

Aprovado: 05/10/2010 